\title{
Geometric Parameterization Technique for Continuation Power Flow Based on Quadratic Curve
}

\section{Alfredo Bonini Neto, Elisabete de Mello Magalhães \& Dilson Amancio Alves}

To cite this article: Alfredo Bonini Neto, Elisabete de Mello Magalhães \& Dilson Amancio Alves (2017) Geometric Parameterization Technique for Continuation Power Flow Based on Quadratic Curve, Electric Power Components and Systems, 45:17, 1905-1917, DOI: 10.1080/15325008.2017.1381203

To link to this article: https://doi.org/10.1080/15325008.2017.1381203

\section{曲 Published online: 23 Jan 2018.}

\section{Submit your article to this journal $\widetilde{ }$}

III Article views: 46

View Crossmark data \lceil 


\title{
Geometric Parameterization Technique for Continuation Power Flow Based on Quadratic Curve
}

\author{
Alfredo Bonini Neto, ${ }^{1}$ Elisabete de Mello Magalhães, ${ }^{2}$ and Dilson Amancio Alves ${ }^{2}$ \\ ${ }^{1}$ School of Sciences and Engineering, São Paulo State University - UNESP, Tupã, Brazil \\ ${ }^{2}$ Electrical Engineering Department, São Paulo State University - UNESP, Ilha Solteira, Brazil
}

\section{CONTENTS}

1. Introduction

2. Continuation Power Flow and Proposed Method

3. Test Results

4. Conclusion

Funding

References
Keywords: continuation power flow, parameterization technique, Lagrange interpolation, $P-V$ curve, loading margin, maximum loading point

Received 12 April 2016; accepted 27 August 2017

Address correspondence to Alfredo Bonini Neto, School of Sciences and Engineering, São Paulo State University - UNESP, Tupã, SP, 17602-496, Brazil. E-mail: bonini@tupa.unesp.br

Color versions of one or more of the figures in the article can be found online at www.tandfonline.com/uemp.

\begin{abstract}
The electricity sector, especially in emerging countries, has experienced several transformations, mainly resulting from the increase of electricity demand. This encourages more investment in the generation sector and causes increasing concerns with the development and improvement of tools for static voltage stability analysis of electrical power systems. This paper presents a new geometric parameterization technique for continuation power flow (CPF) that works based on the addition of a parabola that passes through three points in the plane formed by the variables of total real power losses and loading factor. This technique eliminates the Jacobian matrix singularity at the maximum loading point, which allows obtaining the solution trajectory ( $P-V$ curve) without any need to change the parameter, which is a very common procedure in the currently available CPFs. Intending to define a simple and efficient step size control procedure, the total real power losses values are normalized by its base case value. The results obtained by applying the proposed technique to the IEEE-300 bus system and two real large systems of 638 and 787 buses show its effectiveness.
\end{abstract}

\section{INTRODUCTION}

Over the years, the electricity market, in general, has experienced a series of reforms and restructurings among which the privatization of state-owned electricity industries, which has made an enhancement in the competition among the companies of the sector, can be highlight [1]. This process of transformation has motivated the electric sector to invest in the search for an improvement in the electrical generation, transmission and distribution systems. As a direct consequence, the tools of static voltage stability analysis are getting more attention, especially the continuation power flow (CPF) method [2]-[11]. Nowadays, the CPF is one of the commonly used methods to compare the planning strategies and to analyze several operating conditions of electric power systems, as it allows obtaining the maximum loading point (MLP) and the complete tracing of $P-V$ curves of both the transmission [3] and distribution systems [12], [13]. These curves are widely used for assessment of static voltage stability because they 
facilitate both detection and comprehension of the characteristics of the load. The continuation method has commonly defined in the literature via four basic elements: a parameterization procedure, a predictor step, a step size control strategy, and a corrector step [2], [3], [7]. The parameterization not only provides a way to identify each solution along the solution trajectory to be obtained but also removes the matrix singularity at the MLP. The local parameterization [2] and geometrical parameterization [3]-[12] are the most used parameterization techniques. The use of local parameterization technique in the $\mathrm{CPF}$ was presented in [2]. Arc length parameterization that uses the arc length along the $P-V$ curve tracing was presented in [3]. In the CPF presented [12], which is used for tracing the $P-V$ curve of general unbalanced distribution systems, the arc length, pseudo-arc length, and local parameterization schemes were implemented. The predictor step and step size control strategy are used to find an estimate for the next solution. The corrector step is necessary to correct the approximate solution, in order to avoid error accumulation. The CPF is a time-consuming process due to the extensive number of operating conditions that must be analyzed. For this reason, a large number of works have been proposed in the literature, intending to improve and simplify the CPF algorithm and speed up the $P-V$ curve tracing process.

Many studies on the CPF method have been developed, in which the use of interpolating polynomial is frequent [14]-[18]. A very common application is based on the approximately quadratic curvature presented by the power flow (PF) solution trajectory ( $P-V$ curve) close to the MLP, particularly the critical bus [14]-[16]. In these methods, a quadratic approximation for the $P-V$ curve is used to estimate the power system load margin and voltage magnitudes. In [14], a method derived from the generalized curve fitting approach was applied to estimate the loading margin for each branch outage contingency and ranking them according to their severity. In [15], to improve the accuracy of load margin estimation, a sensitivity-based scheme determines for each contingency, an adaptive step size that is used to compute the second PF solution. A method that uses only PF solution is proposed in [16], it is also based in the approximately quadratic curvature of $P-V$ curve. In several other implementations of CPF, non-linear predictors are developed based on the polynomial interpolation and are used to predict the next $P-V$ curve solution [17], [18]. A good predictor provides points more close to the correct solutions and consequently a fewer iterations will be needed to obtain the exact solutions. The use of the second-order and third-order-based non-linear predictors in these methods are justified by the increase in efficiency resulting from the more accurate predicted points. A very comprehensive work on the use of linear and non-linear predictors can be found in [17], [18].
This paper proposes a new technique for the CPF, which is also based on Lagrange's interpolation method. The Lagrange interpolating polynomial is chosen because it can be determined without any need of solving a system of simultaneous equations [20]. Nonetheless, the proposed technique is not used in the predictor step, but rather in the corrector step. Lagrange's polynomial interpolation formula is not used to predict a next solution using a non-linear predictor or to estimate the MLP using a curve-fitting technique, but to obtain a parameterized equation of quadratic function that passes through three points at the plane defined by total real power losses and load factor, which will be added to the PF equations. The choice of a second-order polynomial for interpolating is based on the quadratic characteristic of the total real power losses' curve as a function of loading factor. The new set of equations is solved by a Newton method to obtain the MLP and to trace the solutions trajectories of the PF equations, i.e., the $P-V$ curves of electric power systems. Besides, a normalization procedure is also proposed, which allows using the same step size for the whole $P-V$ and for all the electric power systems analyzed.

Some tests were performed to clarify and to evaluate the performance of the proposed method. Simulation results are shown for the IEEE-300 bus system and for two realistic systems of 638 and 787 buses, corresponding to parts of South-Southeast Brazilian system. The results show that the proposed CPF method can determine the MLP with the desired precision without any numerical difficulties and with no need of exchange of parameter during the whole tracing process of $P-V$ curve.

\section{CONTINUATION POWER FLOW AND PROPOSED METHOD}

The basic principle of the method is to find the solutions of the set of non-linear algebraic equations that represent the electric power system in the PF problem. A standard procedure for obtaining $P-V$ curves is to modify the set of equations of conventional $\mathrm{PF}$ by adding a parameter that is used for changing the load and generation, in a pre-specified direction [2], [3]. The computation of many operation points enables determination of the MLP with higher precision, however, at a high computational cost. The set of equations of the CPF, in its most general form, is presented as follows:

$$
\mathbf{G}(\boldsymbol{\theta}, \mathbf{V}, \lambda)=\mathbf{0},
$$

which may be rewritten as

$$
\begin{aligned}
\Delta \mathbf{P}(\boldsymbol{\theta}, \mathbf{V}, \lambda) & =\mathbf{P}^{\mathrm{sp}}(\lambda)-\mathbf{P}(\boldsymbol{\theta}, \mathbf{V}) \\
& =\lambda\left(\mathbf{P}_{\mathbf{g}}^{\mathrm{sp}}-\mathbf{P}_{\mathbf{c}}^{\mathbf{s p}}\right)-\mathbf{P}(\boldsymbol{\theta}, \mathbf{V})=\mathbf{0}
\end{aligned}
$$




$$
\begin{aligned}
\Delta \mathbf{Q}(\boldsymbol{\theta}, \mathbf{V}, \lambda) & =\mathbf{Q}^{\mathbf{s p}}(\lambda)-\mathbf{Q}(\boldsymbol{\theta}, \mathbf{V}) \\
& =\left(\mathbf{Q}_{\mathrm{g}}-\lambda \mathbf{Q}_{\mathbf{c}}^{\mathrm{sp}}\right)-\mathbf{Q}(\boldsymbol{\theta}, \mathbf{V})=\mathbf{0}
\end{aligned}
$$

where

$\lambda$ loading factor;

$\mathbf{V}$ and $\boldsymbol{\theta}$ respective vectors of the nodal voltage magnitudes and phase angles, respectively;

$\mathbf{P}^{\text {sp }}(\lambda)$ vector of the difference between the vectors of generated $\left(\mathbf{P}_{\mathbf{g}}^{\mathbf{s p}}\right)$ and consumed $\left(\mathbf{P}_{\mathbf{c}}^{\mathbf{s p}}\right)$ real power specified for the load $(P Q)$ and generation $(P V)$ buses; and

$\mathbf{Q}_{\mathbf{c}}^{\mathbf{s p}}(\lambda)$ vector of consumed reactive power specified for the $P Q$ buses.

Equation (1) assumes that the network loading is proportional to the base case and considers the constant power factor. $\mathbf{P}^{\mathbf{s p}}(\lambda)$ and $\mathbf{Q}^{\mathbf{s p}}(\lambda)$ can also be defined as being equal to $\lambda\left(\mathbf{k}_{\mathrm{Pg}} \mathbf{P}_{\mathbf{g}}^{\mathbf{s p}}+\mathbf{k}_{\mathrm{Pc}} \mathbf{P}_{\mathbf{c}}^{\mathbf{s p}}\right)$ and $\lambda \mathbf{k}_{\mathrm{Qc}} \mathbf{Q}_{\mathbf{c}}^{\text {esp }}$, respectively. The vectors $\mathbf{k}_{\mathrm{Pg}}, \mathbf{k}_{\mathrm{Pc}}$, and $\mathbf{k}_{\mathrm{Qc}}$ are fixed parameters, used to characterize a specific load scenario.

The $P-V$ curve tracing can be performed by successive solutions of Eq. (2) using a PF. In this case, $\mathbf{V}$ and $\boldsymbol{\theta}$ are dependent variables, while $\lambda$ is independent variable, i.e., $\lambda$ is considered as a parameter. Its value is gradually increased from the base case $(\lambda=1)$, until a value for which no solution is obtained. i.e., for which the PF iterative process diverge or takes longer time to find a solution. In general, at this point, there will be a step size control consisting of a simple reduction in the increment (step-length) of $\lambda$ and the new solution is obtained from the last converged solution. The MLP is considered the last point converged after successive repetitions of this procedure. However, the divergence of the PF is a consequence of the singularity of Jacobian matrix (J) of Eq. (2) in MLP, and therefore its value cannot be determined precisely. In order to overcome the numerical difficulties resulting from the $\mathbf{J}$ singularity and allow the determination of the MLP, different implementations of the continuation method were proposed [2]-[6]. In the CPF, $\lambda$ is considered as a dependent variable, and then changed automatically. In this case, Eq. (2), whose dimension is $n=2 n_{P Q}+n_{P V}$ (where $n_{P Q}$ and $n_{P V}$ are the number of PQ and PV buses, respectively), has $n$ +1 unknowns, and an additional parameterizing equation is needed. The parameterization not only provides a way to identify each solution along the solution trajectory to be obtained but also removes the matrix singularity at the MLP. In the context of tracing $P-V$ curve, adding parameterizing equations has become a standard procedure used by practically almost all continuation methods to overcome the aforementioned numerical difficulties and to make possible an accurate determination of MLP [2]-[6]. Therefore, the goal is to remove the singularity of $\mathbf{J}$ through the addition, by means of an equation, of the information lost with the reduction of the rank of the matrix at MLP.

The CPF method presented in [2] uses the local parameterization technique to remove the singularity of $\mathbf{J}$ at MLP, which consists of changing the parameter close to MLP, from the loading factor to a new chosen variable $p$. A proper choice of the continuation parameter depends on the variable that has the greatest rate of change near a given solution. Thus the chosen variable $p$ can be treated as a new parameter and $\lambda$ is regarded as a dependent variable. In the corrector step, the equation $\left(p-p^{s p}=0\right)$, where $p$ and $p^{s p}$ are respectively the variable selected as the continuation parameter and its predicted value, is appended to Eq. (2). Note that the added equation corresponds to a line equation, which is perpendicular to the axis of the variable used as a parameter [7]. When $\lambda$ is used as parameter the added equation corresponds to a line equation perpendicular to the $\lambda$-axis (abscissas axis). In general, close to MLP the voltage magnitude of a bus $k\left(V_{k}\right)$ is used as parameter, which corresponds to a line equation perpendicular to the $V_{k}$-axis (ordinate axis). Therefore, the exchange of variables corresponds to a rotation of $90^{\circ}$ in the diagram $V_{k}$ versus $\lambda$ [7]. In general, the equation to be added may be written as

$$
\mathrm{R}(p, \lambda, \alpha, \beta)=\alpha\left(\lambda-\lambda^{0}\right)-\beta\left(p-p^{0}\right)=0,
$$

where $\alpha$ and $\beta$ are angular coefficients that define the line to be used. The repeated conventional PF approach corresponds to a continuation method with a modified zero-order predictor or trivial predictor [3]. This predictor uses the current solution and a fixed increment in the parameter $\left(\lambda, V_{k}\right.$, or $\left.\theta_{k}\right)$ as an estimate for the next solution. When a probable MLP is approached, the process diverges or takes longer time to find a solution, and the step size is reduced. In the local parameterization technique proposed in [2] the process starts by using $\lambda$ as parameter, then $\alpha=1$ and $\beta=0$, and its value is gradually increased from the base case $\left(\lambda^{0}=1\right)$ up to a value for which a solution can no longer be found. In other words, the iterative CPF process does not converge or diverge. Near the MLP, the parameter is replaced and the variable with the largest variation is chosen as the new parameter, that is, $V_{k}$ or $\theta_{k}$ and $\beta=$ 1 and $\alpha=0$, when $\lambda$ is considered as a dependent variable. After a few points of the curve, it returns back to $\lambda$. However, as the curvature of solutions path is not known initially, an approach to define the parameter changes during the computation process is needed. In [2], it is needed to identify which voltage magnitude is most appropriate to be used as parameter to obtain the MLP. In the CPF presented in [8], the singularity of the $\mathbf{J}$ matrix is avoided by the addition of a line equation, which passes through a chosen point $\left(\lambda^{0} ; V_{k}^{0}\right)$ in the 
plane determined by the bus voltage magnitude $\left(V_{k}\right)$ and loading factor $(\lambda)$ variables:

$$
R\left(V_{k}, \lambda, \alpha\right)=\alpha\left(\lambda-\lambda^{0}\right)-\left(V_{k}-V_{k}^{0}\right)=0,
$$

where $\alpha$ is the angular coefficient of the line. As a new equation is added, $\lambda$ can be treated as a dependent variable and $\alpha$ is considered as the continuation parameter. A trivial predictor with a fixed increment in the parameter $\alpha$ can be adopted. The CPF presented in [6], that uses a line equation passing through a chosen point $\left(\lambda^{0} ; P a^{0}\right)$ in the plane determined by the total real power losses $(P a)$ and loading factor $(\lambda)$, is a global parameterization technique. The technique that use the arc length [3] and those presented in [4]-[6], [8], [11] are other examples of the global parameterization techniques. In the method presented in [6], unlike that proposed in [2], there is no need to change the parameters throughout the $P-V$ curve tracing. Even though sometimes it is necessary to change the set of lines (from one set of lines to another), the method proposed in [6] has an advantage that, unlike the method proposed in [2], the next parameter is known in advance.

To trace the $P-V$ curve for obtaining the MLP, and consequently the loading margin, in the proposed continuation power flow (PCPF) a quadratic term is considered to be added in Eq. (2). This quadratic term is a polynomial function of the second degree that passes through three points in the plane defined by the loading factor $(\lambda)$ and total real power losses $(P a)$ :

$$
\begin{aligned}
\Delta W(\boldsymbol{\theta}, \mathbf{V}, \lambda, \alpha) & =P a(\boldsymbol{\theta}, \mathbf{V})-\left(a(\lambda+\alpha)^{2}+\mathrm{b}(\lambda+\alpha)+c\right) \\
& =0
\end{aligned}
$$

where $\operatorname{Pa}(\boldsymbol{\theta}, \mathbf{V})$ is computed by

$$
P a(\boldsymbol{\theta}, \mathbf{V})=\sum_{k, m \in \Omega} g_{k m}\left(V_{k}^{2}+V_{m}^{2}-2 V_{k} V_{m} \cos \theta_{k m}\right),
$$

where

$\Omega$ set of all network buses, $\theta_{k m}=\theta_{k}-\theta_{m}$;

$V_{k}, V_{m}, \theta_{k}$, and $\theta_{m}$ voltage magnitudes and angles at buses $k$ and $m$;

$g_{k m}$ series conductance of the branch between buses $k$ and $m$; $a, b$, and $c$ coefficients of the quadratic function; and

$\alpha$ new variable that determines the new quadratic functions in the plane $P a(\boldsymbol{\theta}, \mathbf{V})$ versus $\lambda$.

The quadratic function is obtained by Lagrange's interpolation method that uses the following formula:

$$
\operatorname{Pn}(x)=\sum_{i=0}^{n} L_{i}(x) f\left(x_{i}\right)
$$

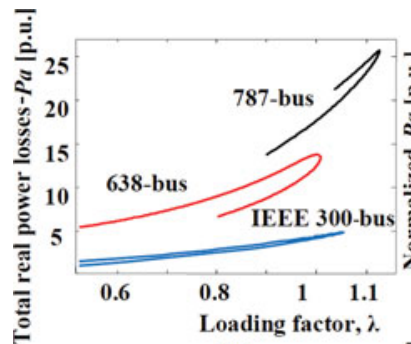

(a)

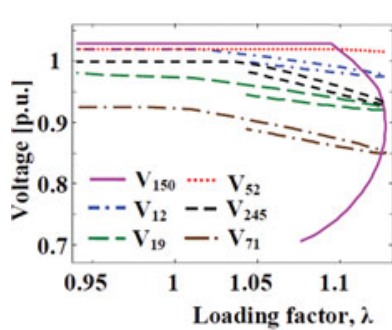

(c)

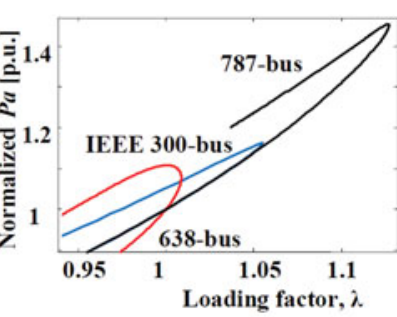

(b)

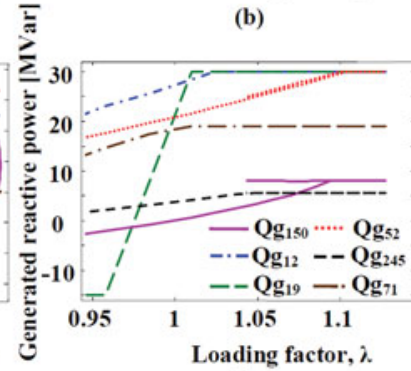

(d)
FIGURE 1. Curves for analyzed systems: (a) $\lambda-P a$ without normalization, (b) $\lambda-P a$ with normalization, (c) $P-V$ curves of some generated bus for the 787-bus system, and (d) the respective generated reactive powers.

where

$$
L_{i}(x)=\prod_{j=0, j \neq i}^{n} \frac{\left(x-x_{j}\right)}{\left(x_{i}-x_{j}\right)}, \quad i=0,1, \ldots, n,
$$

where

$P_{n}(x)$ the Lagrange interpolation polynomial;

$n$ order of polynomial;

$x_{i}$ given point $(i=0,1, \ldots, n)$;

$f\left(x_{i}\right)$ value of the function at $x_{i}$;

$L_{i}(x)$ Lagrange's interpolation coefficient, which is given by Eq. (7).

In Figure 1, the $\lambda-P a$ curves for the three systems studied have been presented. Note that almost all of these curves present a sharp nose, that is, the loading factor and the total real power losses present a simultaneous reversion in its variation tendency, reaching their maximum values at the same point. The upper and lower parts of the curve have practically the same slope, instead of an opposite signal, which means that the curve noses are coincident and both Jacobian matrices are singular at the MLP [10]. Therefore, none of the two magnitudes could be used as a parameter to trace the whole curve because the procedure would fail in the vicinity of their respective maximum point. An extensive study on the limitations of different parameterization methods, including the arc length parameterization, used to trace curves with similar characteristics was presented in [7], [9]-[11]. Despite 
the step size reduction, the CPF fails when these parameterization techniques are used to compute the MLP. However, as shown in Figure 1, all the trajectories of total real power losses as a function of loading factor of the analyzed electric power systems present an approximately quadratic curvature [4]-[6], [9]. Besides, these trajectories present the similar curvature for all operating conditions and are also unaffected by the discontinuities present in the voltage magnitudes which occur due to the discontinuities of the reactive power limits, as shown in Figure 1(c) and (d) for the 787-bus systems. Therefore, the second-degree polynomial (where $P_{n}(x)=$ $a x^{2}+b x+c$ is the quadratic function, being $x$ the loading factor $(\lambda)$ ) seems to be a more suitable choice, which will be confirmed later in the analysis of the systems.

It can be seen from Figure 1(a) that the two axes do not have the same scale, as recommended in [7]. Despite being in per-unit, the numerical values of total real power losses are very different from those of loading factor and can also presents a large variation for different electric power systems. In order to facilitate and simplify the definition of an efficient procedure of control of step size, the total real power losses values are normalized by its base case value. As shown in Figure 1(b), by using the normalization, the values of both variables, $\lambda$ and $P a$, remain within the same range of numerical values. Thus, the new system of equations is written as

$$
\begin{aligned}
\Delta \mathbf{P}(\boldsymbol{\theta}, \mathbf{V}, \lambda) & =\mathbf{P}^{\mathbf{s p}}(\lambda)-\mathbf{P}(\boldsymbol{\theta}, \mathbf{V})=\mathbf{0} \\
\mathbf{Q} \mathbf{Q}(\boldsymbol{\theta}, \mathbf{V}, \lambda) & =\mathbf{Q}^{\mathbf{s p}}(\lambda)-\mathbf{Q}(\boldsymbol{\theta}, \mathbf{V})=\mathbf{0} \\
\Delta W(\boldsymbol{\theta}, \mathbf{V}, \lambda, \alpha) & =\frac{P a(\boldsymbol{\theta}, \mathbf{V})}{P a_{b c}}-\left(a(\lambda+\alpha)^{2}+b(\lambda+\alpha)+c\right) \\
& =0,
\end{aligned}
$$

where the coefficients of the quadratic function, $a, b$, and $c$ are calculated by Eq. (7), and $\alpha$ is the new variable that determines the new quadratic functions. The symbol $P a_{\mathrm{bc}}$ corresponds to the total real power losses value, calculated from the base case. As a new equation is added, $\lambda$ can be treated as dependent variable and $\alpha$ is considered as continuation parameter (value is prefixed). As the number of unknown variables is equal to the number of equation, the necessary condition for the resolution of Eq. (8) is obtained, while the new matrix has maximum rank, i.e., it is not singular. The PCPF uses the modified zero-order polynomial or trivial predictor [2]. For $\alpha=\alpha^{0}+\Delta \alpha$, the solution of (8) will provide the new operating point $(\theta, V, P a, \lambda)$ corresponding to the intersection of trajectory solutions $(\lambda-\mathrm{Pa})$ with the parabola whose new value $\left(\alpha^{0}+\Delta \alpha\right)$ was specified.

\subsection{General Procedure for Tracing the $P-V$ Curve}

As shown in Figure 2(a) and (b), the $P-V$ curve of any bus $k$ in the system is obtained with the corresponding desired

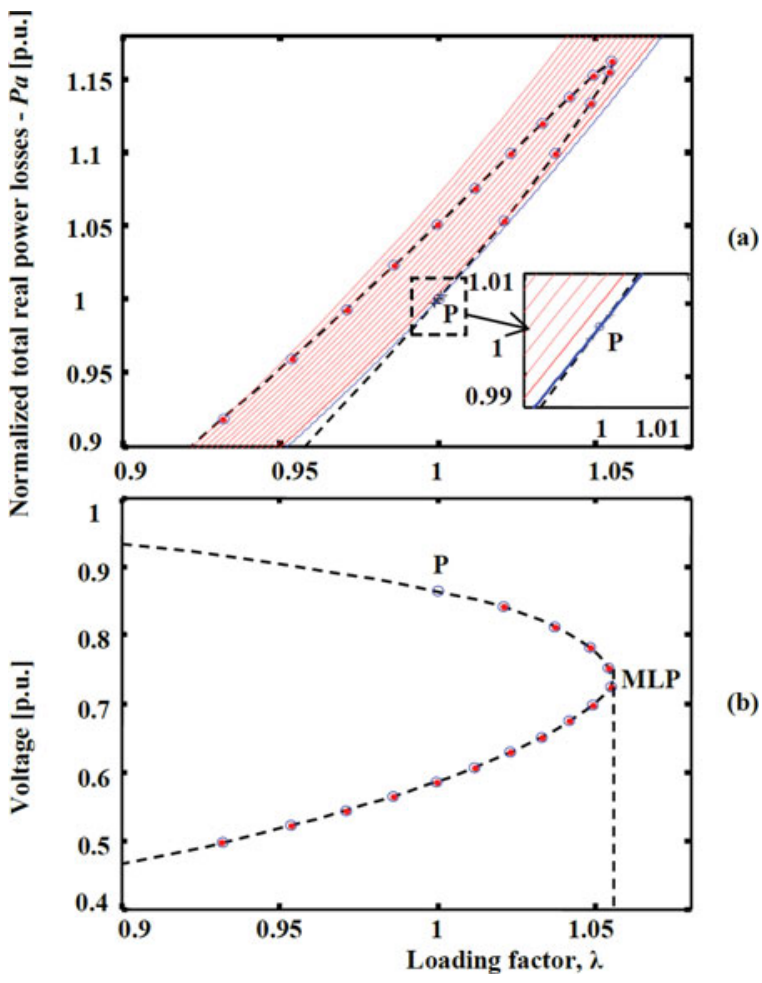

FIGURE 2. (a) Parabolas in the plane $\lambda-P a$ and (b) voltage magnitude of critical bus, determined by the PCPF.

values of voltage magnitude and loading factor, which is stored while obtaining the $\lambda-P a$ curve. The $\lambda-P a$ curve is traced as follows:

Step 1. The three first operating points, including the base case operating point "P," are obtained by using a conventional PF, and by using Eq. (7), the corresponding coefficients of the quadratic function, $a, b$, and $c$, that passes through the three points in the plane defined by the loading factor $(\lambda)$ and total real power losses $(P a)$, are computed, see Figure 2(a).

Step 2. The next points of curve $\lambda-P a$ are obtained by using the PCPF and applying a gradual increment (i.e., with a fixed step) to the continuation parameter $\alpha$, $\alpha^{i+1}=\alpha^{i}+\Delta \alpha$, whose initial value is equal to zero;

Step 3. When $\lambda$ changes its sign, the MLP has been passed. So, if it is desired to determine the MLP more accurately, the solution can be traversed backward with a smaller step size.

In function of the similar curvature presented by the $\lambda-P a$ curve of all the analyzed systems, the PCPF has the advantages of not needing to change the parameter during the complete tracing of the curve, and the use of a single fixed step size for all systems, therefore avoiding the need of successive step size reductions close to the MLP. The adopeted step size was chosen based on the values of the continuation parameter 
$(\alpha)$ calculated with the $\lambda$ and $P a$ stored values while obtaining the $P-V$ curve using the voltage magnitude of the critical bus. A fixed step size of 0.01 p.u. was commonly adopted when the voltage magnitude of the critical bus is used as a parameter. For the step size of 0.01 p.u. in the voltage magnitude, a step size of approximately 0.001 p.u. was found for $\alpha$.

\section{TEST RESULTS}

This section discusses the results of applying the PCPF method for the IEEE 300-bus and two systems corresponding to parts of the south-southeast Brazilian system with 638-bus and 787-bus. The IEEE 300-bus test case has been developed by the IEEE Test Systems Task Force under the direction of Mike Adibi in 1993 [19]. The Brazilian 638- and 787-bus systems correspond to part of the Brazilian national interconnected system. The 638-bus system contains 98 generator buses, 1,050 branches and 540 load buses, while the 787 bus system contains 112 generator buses, 1,109 branches and 675 load buses. For all tests, the tolerance adopted for mismatches is $10^{-5}$ p.u. The reactive power limits $(Q)$ in PV buses are the same as those used in the conventional PF. In each iteration, the reactive generations at all $\mathrm{PV}$ buses are compared to their respective limits. In case of violation, a PV bus is switched to $\mathrm{PQ}$. This bus can be switched back to PV in future iterations. The loads are modeled as constant power and the parameter $\lambda$ is used to simulate the increments of real and reactive loads, considering constant power factor. Each load increase is followed by an increase of the equivalent generation, using $\lambda$. The purpose of the tests is to show the efficiency and robustness of the proposed methods to trace the $P-V$ curve of electrical power systems.

\subsection{Results of the PCPF for the IEEE 300-bus System}

Figure 3 shows the results of the proposed method for the IEEE 300-bus system, i.e., considering the equation of a quadratic function located in the plane of $\lambda-P a$ and $\alpha$ as the continuation parameter. Figure 3(a) shows the curve of total real power losses versus the loading factor. The curve tracing process starts from the base case $(\lambda=1)$ with getting the first three points by a CPF or a conventional PF. The other points on the $\lambda-P a$ curve can be determined through successive solutions of Eq. (8) and adopting a fixed step size for the value of parameter $\alpha$. The step-length $(\Delta \alpha)$ adopted to obtain the other points on the curve was 0.002 for all electric power systems. This value was adopted in order to calculate a few points along the curve.

From Figure 3(a), it can be seen that the lower and upper parts of the $\lambda-P a$ curve have practically the same slope,

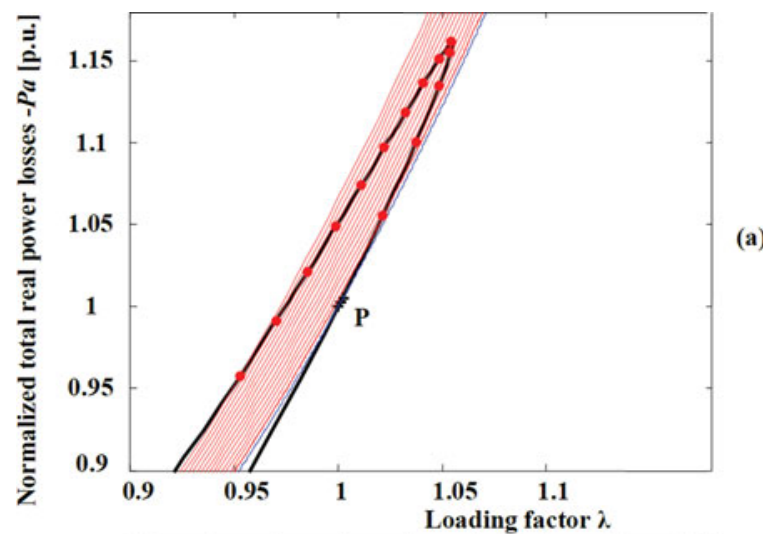

(a)
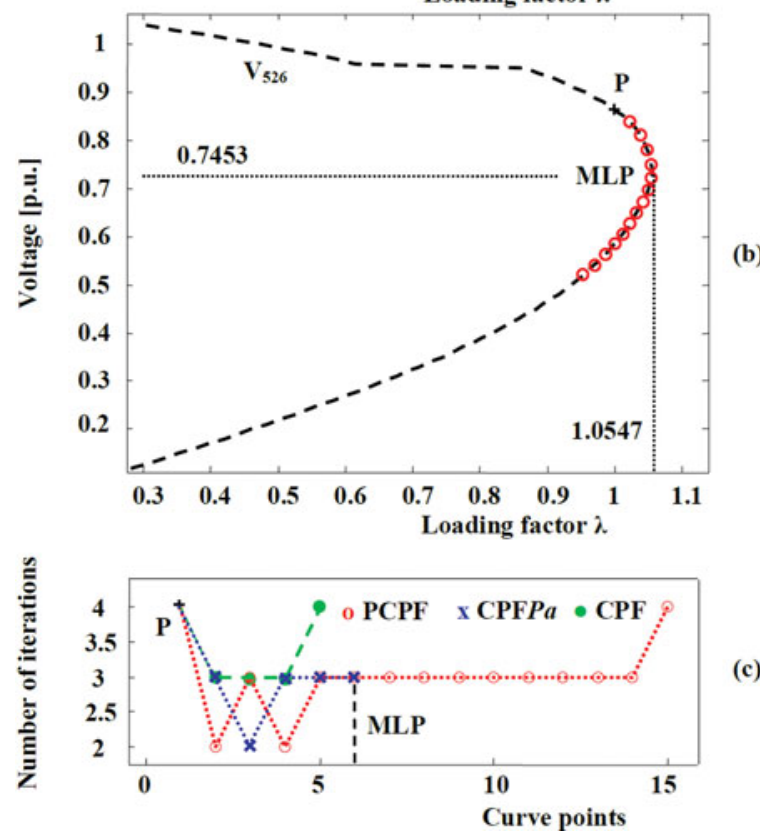

(c)

FIGURE 3. Performance of the PCPF for the IEEE 300-bus system: (a) $\lambda-P a$ curve, (b) $P-V$ curve of the critical bus 526 , and (c) number of iterations.

instead of an opposite signal. In such cases, the loading factor and total real power losses showed a simultaneous reversal in its variation tendency, that is, the noses are coincident. This means that both Jacobian matrices of the CPF, the one using loading factor or the total real power losses, are singular at the MLP. Thus, these variables cannot be used as parameters to obtain the MLP because the method will present numerical difficulties in their neighborhood. Moreover, as it can be seen from Figure 3, the PCPF allows the determination of the MLP without the numerical problems related to Jacobian matrix singularity.

Figure 3(b) shows the $P-V$ curve obtained by storing, during the tracing of the curve $\lambda-P a$, the corresponding desired values of the voltage magnitude $\left(V_{526}\right)$ and $\lambda$. The values of 


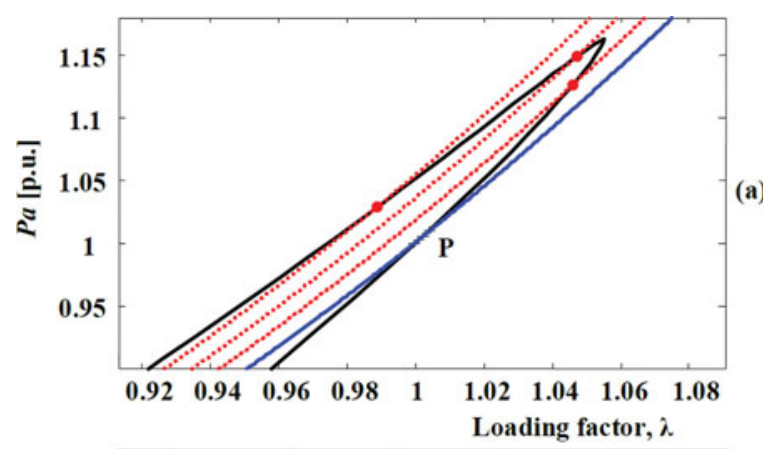

(a)
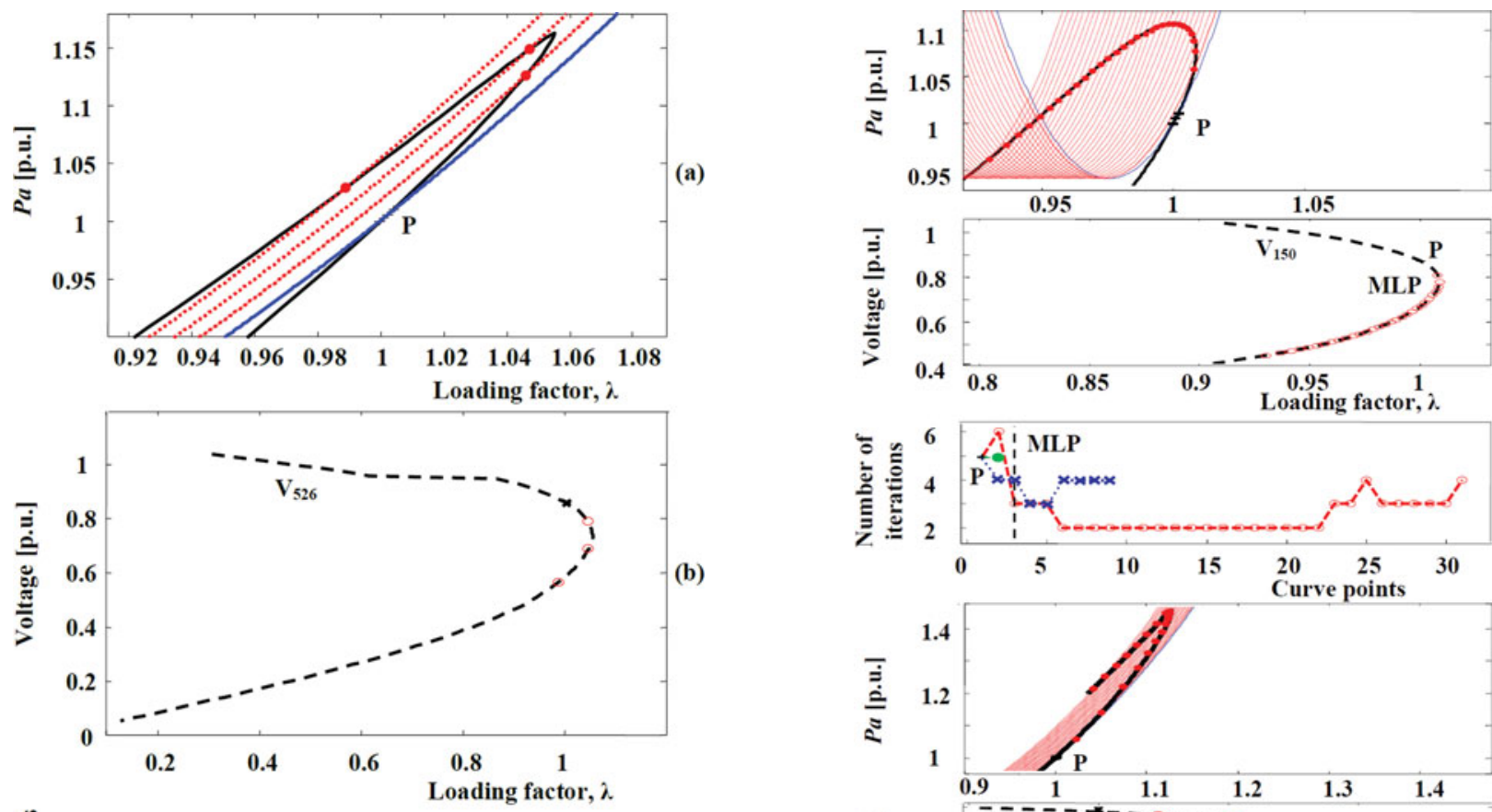

(a)

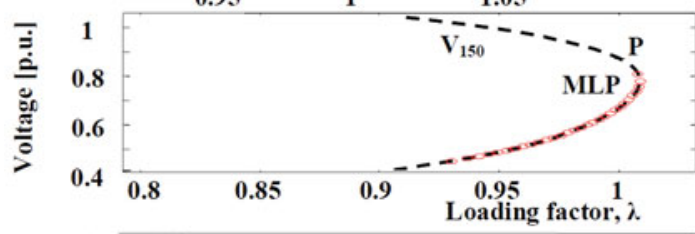

(b)
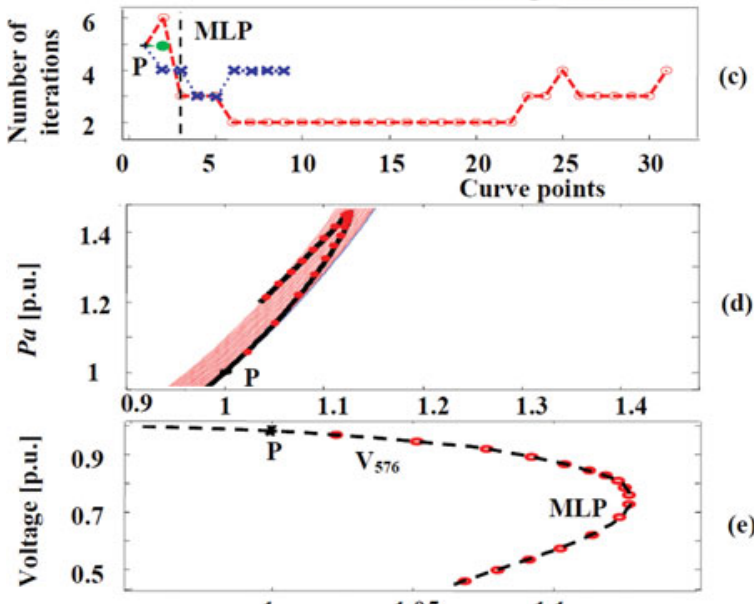

(d)

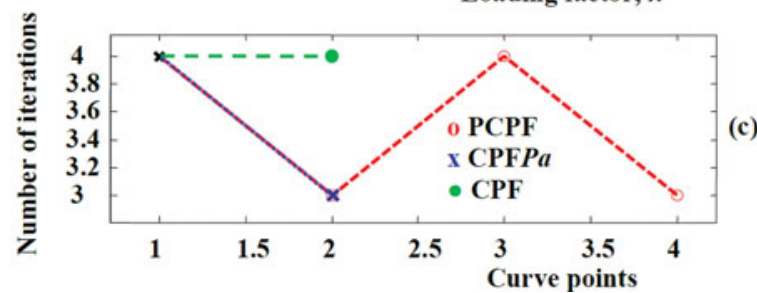

FIGURE 4. Performance of the PCPF for the IEEE 300-bus system: (a) $\lambda-P a$ curve, (b) $P-V$ curve of the critical bus 526 , and (c) number of iterations with quadruplicate step, $\Delta \alpha=$ 0.008 .

the $\lambda$ and $V_{526}$ in the MLP are 1.0547 p.u. and 0.7453 p.u., respectively.

Figure 3(c) shows the number of iterations used to obtain the curve. It can be concluded that the proposed method succeeds in finding each points of the curve, including the MLP, with a small number of iterations and without changing the parameter. Also note that an automatic step size control occurs around the MLP, even using a fixed step size $(\Delta \alpha)$. This gives the additional advantage of providing a larger number of points in the MLP region automatically.

It is important to point out that the method is not so sensitive to the adopted step size of 0.002 . The possibility of using large step sizes is another important point, once a reduction in the number of points of the $P-V$ curve is provided. In general, the continuation methods demand a good step size control in order to avoid ill-conditioning and numerical problems, otherwise it can lead to divergence. Intending to demonstrate the robustness of the PCPF, Figure 4 presents the results for the same case of Figure 3, but considering a quadruplicate

step size $(\Delta \alpha=0.008)$. Note that even quadruplicating the step size, the method allows the complete tracing of the curve and still keeps a low number of iterations for the solution of each point, as shown in Figure 4(c).

\subsection{Results of the PCPF for Real Larger Systems}

Figure 5 shows the results of the PCPF for the 638 and 787 bus systems; these systems are corresponding to parts of the South-Southeast Brazilian system, which are heavily loaded, particularly the 638-bus that is a system under a very stressed loading condition with the MLP equal to 1.0087 p.u., i.e., an operating point very close the base case.

Figure 5(a) and (d) shows the $\lambda-P a$ curves, while Figure 5(b) and (e) presents the respective $P-V$ curves of critical bus $\left(V_{150}\right.$ and $\left.V_{576}\right)$ of each system obtained by storing, 

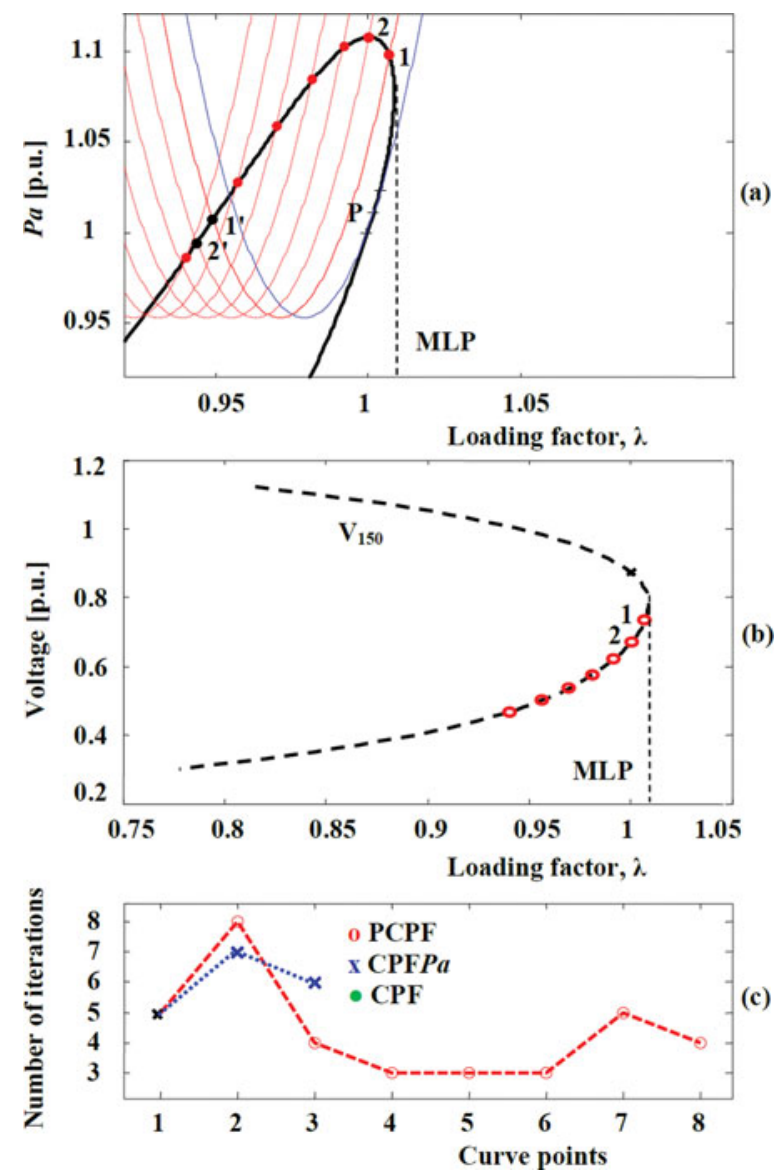

FIGURE 6. Performance of the PCPF for the 638-bus system: (a) $\lambda-P a$ curve, (b) $P-V$ curve of the critical bus 150 , and (c) number of iterations with quadruplicate step size, $\Delta \alpha$ $=0.008$.

during the tracing of the $\lambda-P a$ curve, the corresponding values of the voltage magnitude and loading factor. Figure 5(c) and (f) presents the number of iterations used to obtain each point of the curves. Note that the PCPF also succeeds to find the MLP and providing the correct solutions for the lower and upper parts of the curve with a reduced number of iterations.

Figures 6 and 7 present the results of the PCPF for the 638-bus and 787-bus systems, respectively, solving the same problems of Figure 5 with quadruplicate step size. The respective numbers of iterations for each point is still small, as shown in Figures 6(c) and 7(c). In Figure 7(e), one can be seen the number of iteration needed to compute each point with an increased step size of 0.012 . Also for these systems the method worked very well with no difficulties for tracing the whole $P-V$ curve, even when considerably large step sizes were used. It can also be seen from Figure 6(a) that for each parabola the intersection of the trajectory solutions $(\lambda-P a)$ with the parabola yields two solutions (e.g., 1 and $1^{\prime}$, and 2 and $2^{\prime}$ ). It can be seen from Figure 6(a) that by changing the
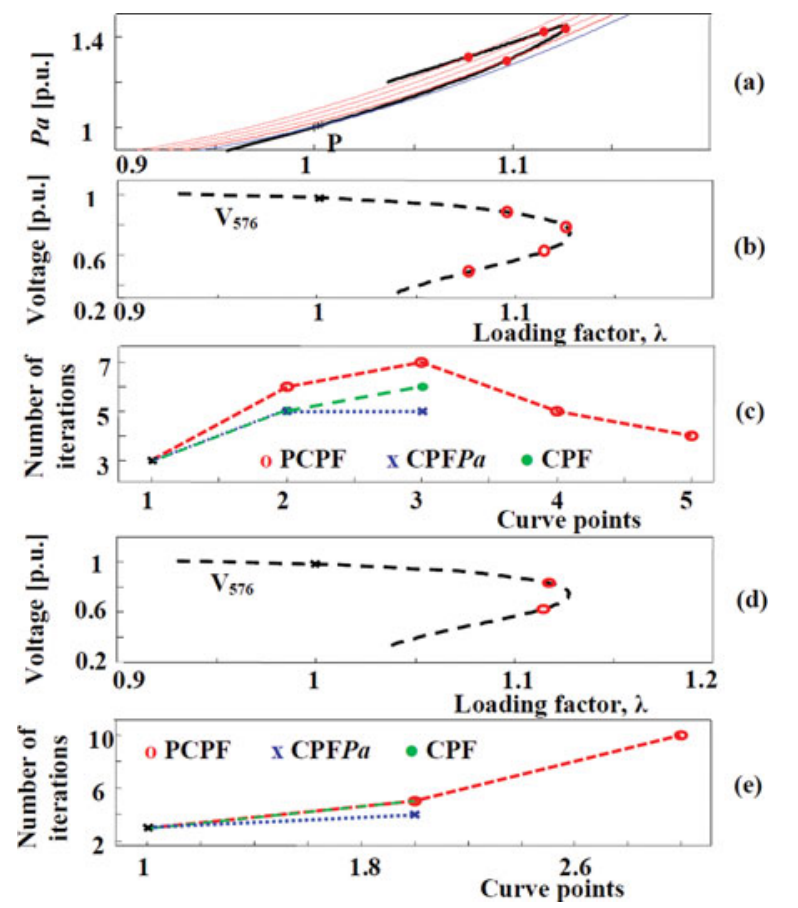

FIGURE 7. Performance of the PCPF for the 787-bus system: (a) $\lambda-P a$ curve, (b) $P-V$ curve of the critical bus 576 with quadruplicate step size, $\Delta \alpha=0.008$, (c) number of iterations with $\Delta \alpha=0.008$, (d) $P-V$ curve of the critical bus 576 with sextuplicate step size, $\Delta \alpha=0.012$, and (e) number of iterations with $\Delta \alpha=0.012$.

parameter $\alpha$, it was possible to compute the point 1 from the previous solution of base case $(\mathrm{P})$, and from point 1 the next one, point 2. In Figure 6(b), it can be seen the corresponding voltage magnitude values that were stored while obtaining the $\lambda-P a$ curve. The local convergence characteristic of Newton's method guarantees that the system converges to the closest solution points 1 and 2 instead of $1^{\prime}$ and $2^{\prime}$. When passing through the MLP, there is a sign change of $\Delta \lambda$, which allows not only its determination but also distinguishing a stable solution from an unstable. Point 1 is after the MLP, and so if a more precise MLP value is required, it is only necessary to go back to previous point and then reduce the step size $(\Delta \alpha)$.

\subsection{Performance Comparison of the PCPF with Diferent Parameterizations}

The objective of the above test is to compare the performance (number of iterations) between the PCPF and the ones presented in [4], [5], parameterized by the total real power losses $(P a)$, and parameterized by loading factor $(\lambda)$ or by the voltage magnitude of the respective critical buses $(V)$, during the changes from one state to another. Thus, the corresponding parameters $(\mathrm{Pa}, \lambda, V)$ of each state were obtained using the PCPF. A fixed step size of 0.002 was used. Once all the states 


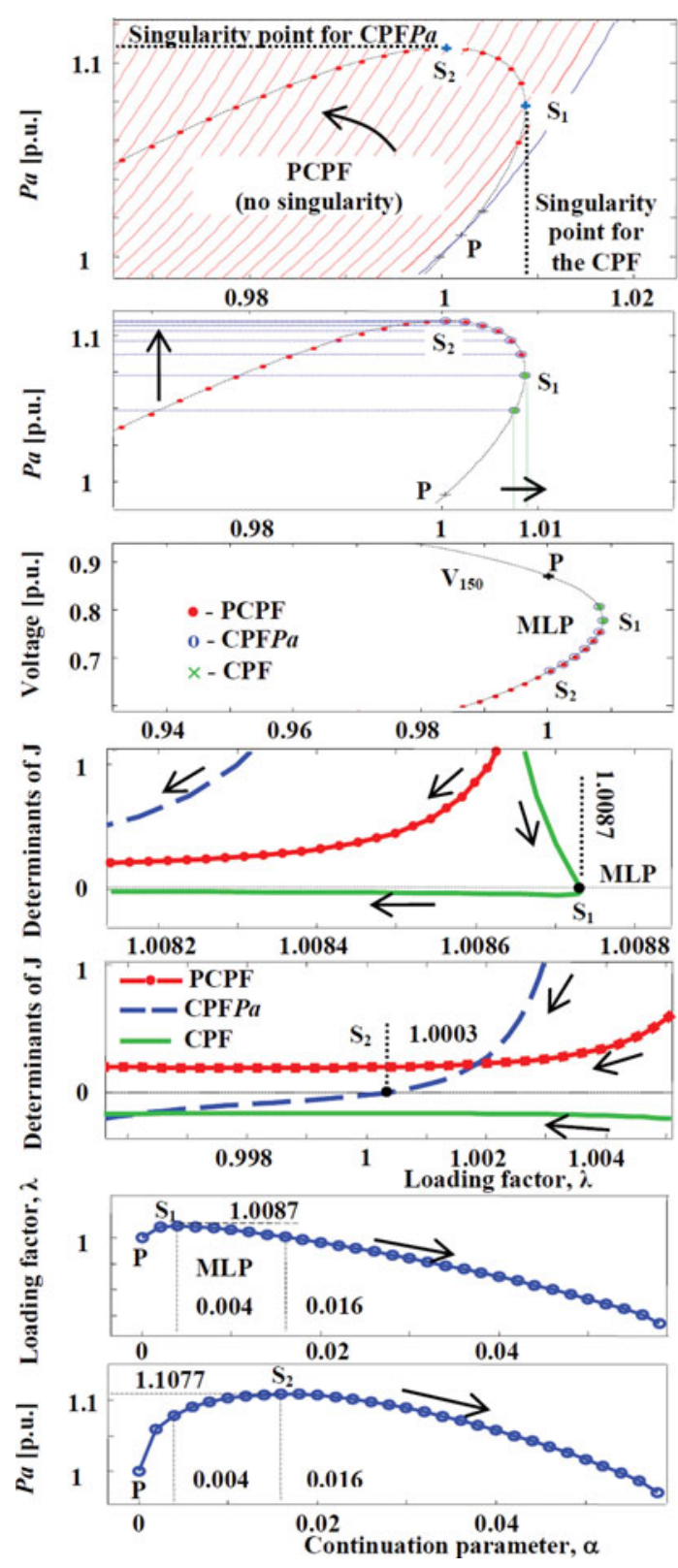

(a)

(b)

(c)

(d)

(e)

(f)

(g)

FIGURE 8. Comparison of the methods for the 638-bus system: (a) parameterization based on quadratic curves in the $P a$ curve, (b) performance of the methods parameterized by $P a$ and $\lambda$, (c) results in the $P-V$ curve, (d) normalized determinants around the MLP region (point $S_{1}$ ), and (e) normalized determinants around the maximum total real power losses region (point $\mathrm{S}_{2}$ ), (f) $\lambda$ as function of the new parameter $\alpha$, (g) $P a$ as function of the new parameter $\alpha$.

were obtained, the respective stored parameters are used as estimated by respective methods, and by using the trivial predictor. The parameter values obtained in this way ensure that the system is taken from the same initial state to the same final state. Figures 8 and 9 provide a fair comparison between the performance of the methods, for the 638-bus and 787-bus
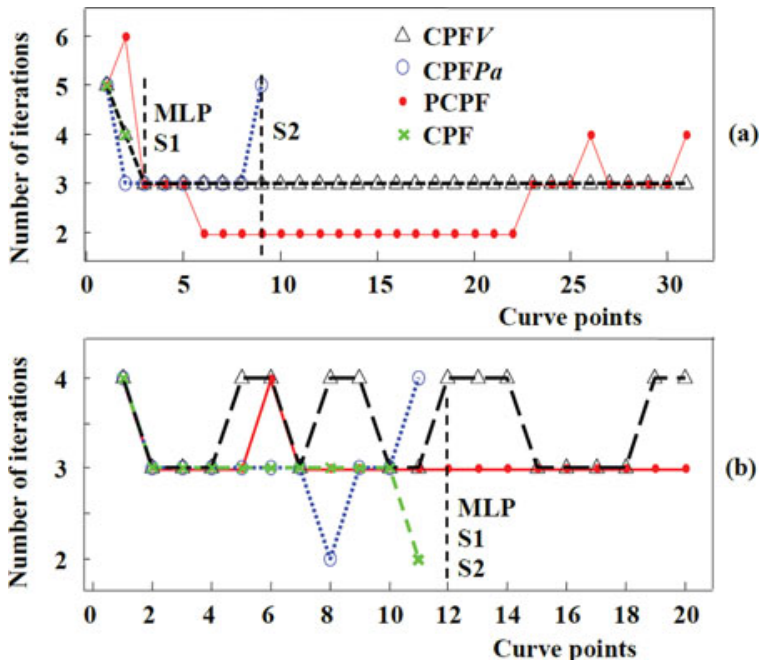

FIGURE 9. Comparison between the performances of the methods: (a) number of iterations for the 638-bus system and (b) number of iterations for the 787-bus system.

systems. It is worth mentioning that, in the Figures 8(b) and (c), after the corrector step, as expected, the corresponding operating points are the same for all the methods.

All points on $\lambda-P a$ curve were computed by using the PCPF and by applying a fixed step size of 0.002 to the continuation parameter $\alpha$. These points are obtained with no ill-conditioning problems and without changing the parameter during the computation process. This is not true for the conventional PF and the CPF parameterized by $\lambda$. Figure 8(d) and (e) shows the normalized determinants of Jacobian matrices. Point $S_{1}$ corresponds to the MLP, i.e., where $\lambda$ reaches its maximum value. Then, if $\lambda$ is used as a parameter, the CPF Jacobian matrix will present a singularity at point $S_{1}$, as shown in Figure 8(d). Note that at this point $\mathrm{S}_{1}$, the conventional PF Jacobian matrix is also singular. So, when the neighborhood of point $S_{1}$ is reached, these methods will present numerical difficulties, the conventional PF fails to obtain a solution and for the CPF parameterized by $\lambda$, a parameter switching will be necessary. In case $P a$ is used as a parameter, the respective modified Jacobian matrix will present a singularity at the point $\mathrm{S}_{2}$, in this case shortly after the MLP. Note from Figure 1(b) that for this parameter is common to find noses that are coincident and therefore both Jacobian matrices are singular at the MLP. However, the MLP and all $P-V$ curves can be easily computed with the proposed parameterized method (PCPF) because the singularity is removed when $\alpha$ is used as a parameter, as shown in Figure 8(f) and (g), which exhibit for the 638-bus system the behavior of the variable $\lambda$ and $P a$ as a function of the new parameter. The good convergence performance in the MLP 


\begin{tabular}{|c|c|c|c|c|c|c|c|c|}
\hline \multirow[b]{2}{*}{ System } & \multicolumn{4}{|c|}{ Voltage Stability Margin (\%) } & \multicolumn{4}{|c|}{ Critical Voltage (p.u.) } \\
\hline & CPF & CPFV & CPFPa & PCPF & $\mathrm{CPF}$ & CPFV & CPFPa & PCPF \\
\hline 300 & 5.45 & 5.52 & 5.52 & 5.53 & 0.7477 & 0.7383 & 0.7383 & 0.7325 \\
\hline 638 & 0.86 & 0.87 & 0.87 & 0.87 & 0.7916 & 0.7780 & 0.7780 & 0.7780 \\
\hline 787 & 12.70 & 12.72 & 12.72 & 12.73 & 0.7616 & 0.7539 & 0.7539 & 0.7458 \\
\hline
\end{tabular}

TABLE 1 . Voltage stability margin and critical voltage of the analyzed systems.

CPF: continuation power flow parameterized by $\lambda, \mathrm{CPF} V$ : continuation power flow parameterized by voltage magnitude of critical bus, CPFPa: continuation power flow parameterized by $P a$, PCPF: proposed continuation power flow parameterized by $\lambda$ with step size 0.001 .

vicinity provided by the PCPF is due to the low degree of nonlinearity of the modified equation system obtained by using the new parameter $\alpha$. Note that when $\alpha$ is used as parameter, the added equation corresponds to a line equation perpendicular to the $\alpha$-axis (abscissas axis). In Figure 8(c), the respective $P-V$ curve obtained for the critical bus 150 can be seen.

Figure 9 provides a comparison of the number of iteration, needed to compute each point, between the PCPF and the different aforementioned parameterization. Table 1 shows for the three analyzed systems the voltage stability margin and the corresponding magnitude voltage of the critical bus, computed by the three proposed parameterization techniques. It can be verified that by using the voltage magnitude of the critical bus as a continuation parameter, the MLP can be obtained whitout the numerical problem related to the singularity of Jacobian matrix. In general, $\lambda$ or a voltage magnitude is used as the continuation parameter. In the local parameterization technique, the $P-V$ curve tracing is started by using $\lambda$ as parameter and close to the MLP it changes to the voltage magnitude that presents the largest variations, in order to overcome the singularity of Jacobian matrix [2]. However, as the curvature of solutions path is not known initially, an approach to define the parameter changes during the computation process is needed. In [2], it is needed to identify which voltage magnitude is most appropriate to be used as parameter to obtain the MLP. On the other hand, with the proposed parameterization, a change in the continuation parameter is not necessary during the whole $P-V$ curve tracing process. Moreover, the overall numbers of iterations when voltage magnitude of critical bus $\left(V_{150}\right)$ is used as the parameter are higher than that of PCPF. They take 13.5 and $11.4 \%$ more than the PCPF for the 638-bus and 787-bus systems, respectively.

\subsection{The Influence of using a Constant Jacobian}

The voltage static stability studies are widely used in the planning and real-time operation to assess the voltage stability margins and to define preventive control actions for several operating conditions, considering often for each, hundreds of contingency cases. In these studies, the main desirable features for the $\mathrm{CPF}$ are robustness, computational efficiency, and effectiveness for obtaining the MLP. It has been proven that the Newton-Raphson algorithm is the most robust approach. The adjustments of solutions as a result of reactive limit violations at generation bus also affect the convergence of the PF methods. Thus, to improve the computational efficiency of the PF methods, a commonly used procedure is to not update $\mathbf{J}$ at every iteration, but only when the system undergoes a significant change (e.g., when bus type switching, from $\mathrm{PV}$ to $\mathrm{PQ}$, is due to reactive limit violations). This procedure, known as the "dishonest Newton method" [11], [21], [22], often enables a considerable reduction in the computing time. Therefore, in this section, the proposed parameterization technique is evaluated comparing their performance by considering two procedures: in the first (P1), the updating of the whole Jacobian matrix is performed at every iteration, and in the second one (P2), only when the system undergoes a significant change (e.g., when a voltage controlled bus (PV) is converted to a load bus (PQ) as a consequence of a violation of one of their reactive limits), or when the number of iterations exceeds a predefined threshold value (eight iterations).

Table 2 presents the results of the updating procedures considering a step size $(\Delta \alpha)$ of 0.002 and a quadruplicate step size of 0.008 . For the 787-bus system, Table 2 is used to compare the performance of the method considering a

\begin{tabular}{|c|c|c|c|c|c|c|}
\hline \multirow[b]{3}{*}{ System } & \multicolumn{2}{|r|}{ P1 } & \multicolumn{3}{|c|}{ P2 } & \multirow{3}{*}{$\begin{array}{c}\text { CPU Ratio } \\
(\%)\end{array}$} \\
\hline & & $\overline{\mathrm{CPU} \text { Time }}$ & & & $\overline{\text { CPU Time }}$ & \\
\hline & IC & (p.u.) & $I C$ & $A C o$ & (p.u.) & \\
\hline 300 & 41 & 1.000 & 51 & 11 & 0.385 & 61.5 \\
\hline 638 & 78 & 1.000 & 182 & 10 & 0.403 & 59.7 \\
\hline 787 & 58 & 1.000 & 92 & 17 & 0.449 & 55.1 \\
\hline $300^{\mathrm{a}}$ & 10 & 1.000 & 13 & 5 & 0.555 & 44.5 \\
\hline $638^{\mathrm{a}}$ & 30 & 1.000 & 43 & 15 & 0.633 & 36.7 \\
\hline $787^{\mathrm{a}}$ & 22 & 1.000 & 26 & 14 & 0.534 & 46.6 \\
\hline $787^{\mathrm{b}}$ & 15 & 1.000 & 16 & 9 & 0.485 & 51.5 \\
\hline
\end{tabular}

TABLE 2 . Performance of the parameterization technique based on quadratic curve for procedures $\mathrm{P} 1$ and $\mathrm{P} 2$.

${ }^{\text {a }}$ Quadruplicate step-size.

${ }^{\mathrm{b}}$ Sextuplicate step-size.

$A C o$ - actualization count. 

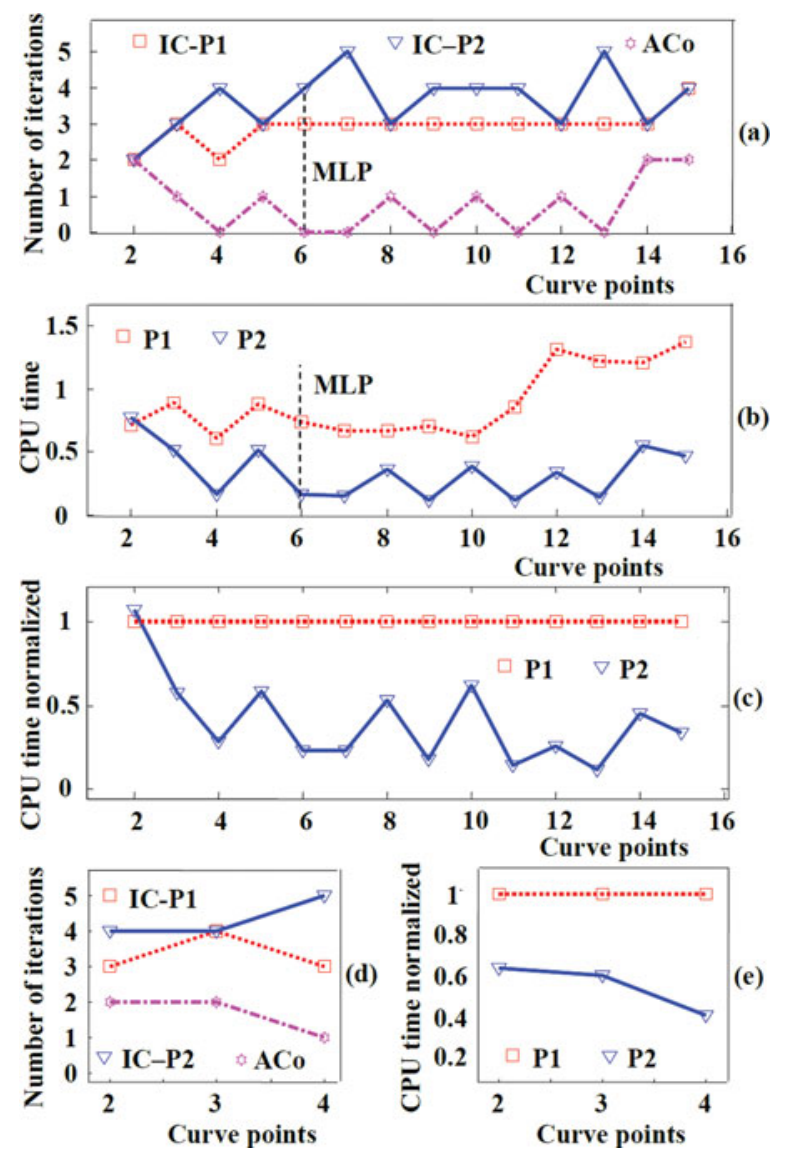

FIGURE 10. Performance of the PCPF for IEEE 300-bus system with $\Delta \alpha=0.002$ : (a) number of iterations for procedures $\mathrm{P} 1, \mathrm{P} 2$ and with actualization count $(A C o)$, (b) CPU time for both procedures, (c) CPU time normalized for both procedures, (d) $\Delta \alpha=0.008$, number of iterations for procedures P1, P2 and with actualization count $(A C o)$, and (e) CPU time normalized for both procedures with $\Delta \alpha=0.008$.

sextuplicate step size $(\Delta \alpha=0.012)$. For both procedures, the total number of iterations (IC) to trace the complete $P-V$ curve is shown, and for $\mathrm{P} 2$, the total number of iterations $(A C o)$ is also shown, for which an updating of the matrix occurs. The CPU time requirement for the PCPF, considering the procedure $\mathrm{P} 2$, is shown in the sixth column of Table 2 . Their values were normalized by the respective $\mathrm{CPU}$ times of the procedure $\mathrm{P} 1$, which is in the third column of the table. As can be seen in the forth column, despite the total number of iterations is larger for the procedure $\mathrm{P} 2$, it is possible to obtain a reduction of the computational time, i.e., an efficiency improvement in the proposed technique, without losing robustness, as can be confirmed in the sixth and seventh columns of the table.

Figure 10 shows the number of iterations and the CPU time needed by the procedures $\mathrm{P} 1$ and $\mathrm{P} 2$, for each point of the $P-V$ curve of the IEEE 300-bus system, presented in Figure 3. Note that, as shown in Figure 10(a), the number of
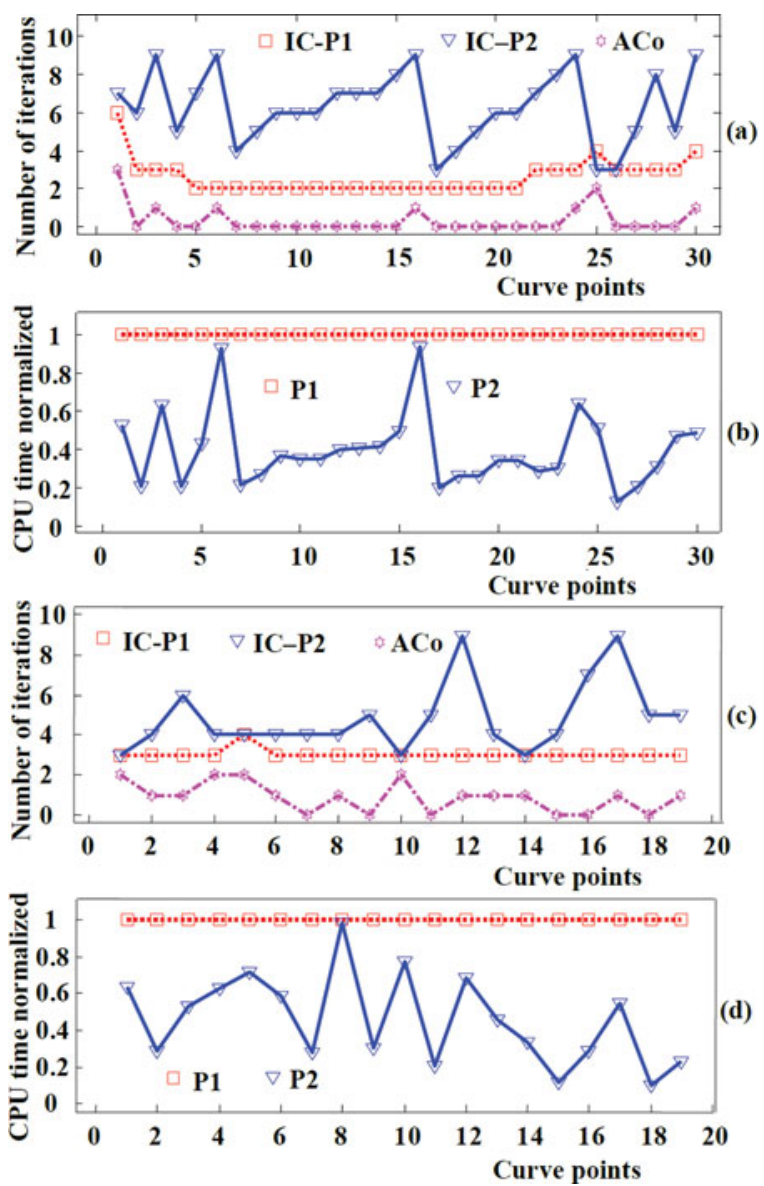

FIGURE 11. (a) Performance of the PCPF for 638-bus system with $\Delta \alpha=0.002$, number of iterations for procedures $\mathrm{P} 1, \mathrm{P} 2$ and with actualization count $(A C o)$, (b) CPU time normalized for both procedures, (c) performance of the PCPF for 787-bus system with $\Delta \alpha=0.002$, number of iterations for procedures $\mathrm{P} 1, \mathrm{P} 2$ and with actualization count $(A C o)$, and (d) CPU time normalized for both procedures with $\Delta \alpha=0.002$.

iterations of the procedure $\mathrm{P} 2$ is $25 \%$ larger than $\mathrm{P} 1$. However, the number of iterations $(A C o)$ for which there is a matrix updating is $73 \%$ lower than for $\mathrm{P} 1$, resulting in a reduction of $61.5 \%$ of the overall CPU time, as shown in Table 2 and Figure 10(b), where the CPU times to compute each point is shown. The results for each point presented in Figure 4, can also be seen in Figure 10. Note from Table 2 that despite of a large step-size of 0.008 , an overall CPU time reduction of $44.5 \%$ is obtained for $\mathrm{P} 2$, compared to the $\mathrm{P} 1$.

Figure 11 shows the numbers of iterations and respective CPU times needed by the procedures $\mathrm{P} 1$ and $\mathrm{P} 2$, for obtaining the solution of each point corresponding to the $P-V$ curves of the 638-bus and 787-bus systems presented in Figure 5. Once again the numbers of iterations of the procedure $\mathrm{P} 2$ are 133 and $58.6 \%$ larger than the $\mathrm{P} 1$, respectively for the 638-bus and 787-bus systems. Moreover, as expected, the respective CPU 

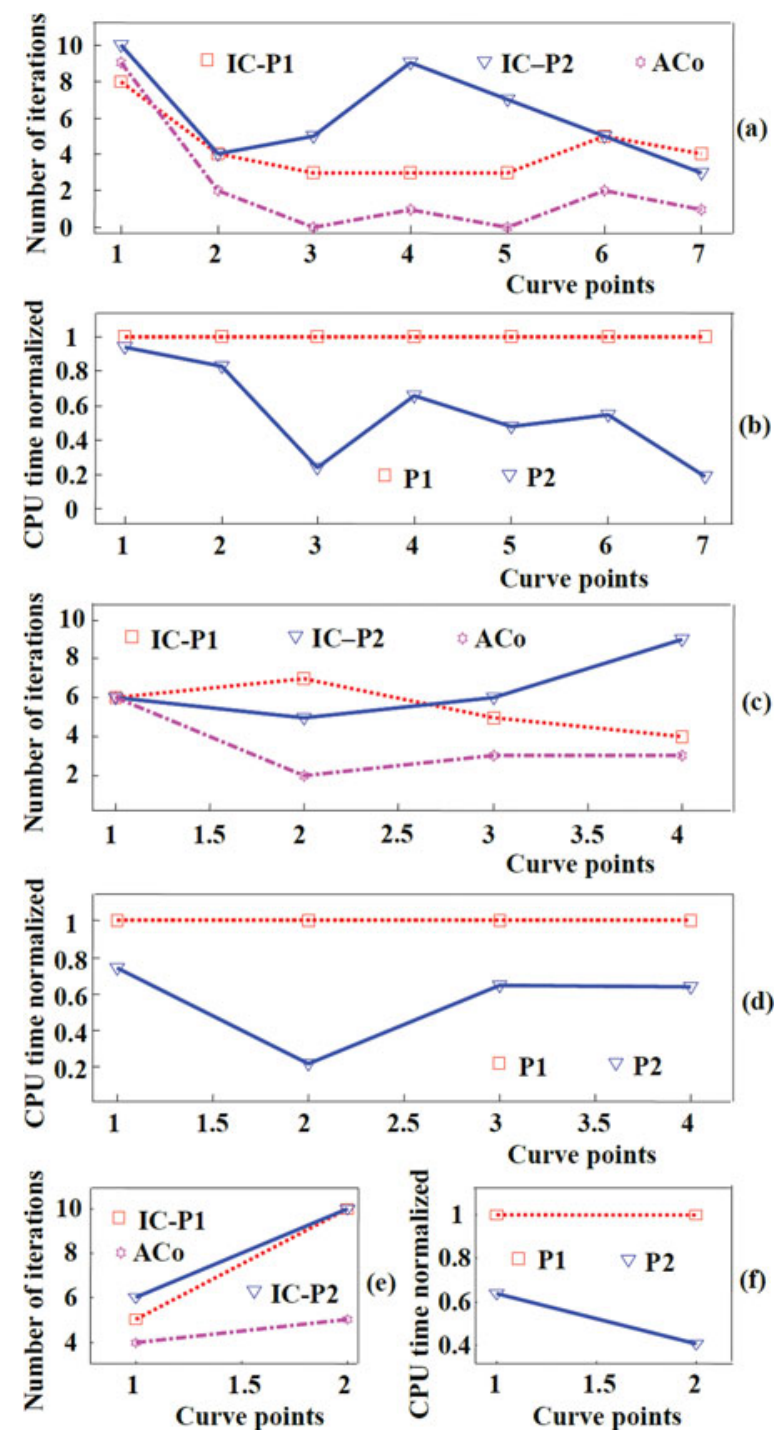

FIGURE 12. Performance of the PCPF for 638-bus system with $\Delta \alpha=0.008$ : (a) number of iterations for procedures $\mathrm{P} 1, \mathrm{P} 2$ and with actualization count $(A C o)$, (b) CPU time normalized for both procedures, (c) performance of the PCPF for 787-bus system, number of iterations for procedures $\mathrm{P} 1$, $\mathrm{P} 2$ and with actualization count $(A C o)$ with $\Delta \alpha=0.008$, (d) CPU time normalized for both procedures with $\Delta \alpha=0.008$, (e) number of iterations for procedures P1, P2 and with actualization count ( $A C o$ ) with $\Delta \alpha=0.012$, and (f) CPU time normalized for both procedures with $\Delta \alpha=0.012$.

time reductions are 59.7 and $55.1 \%$ lower, as can be confirmed in Table 2 and Figure 11(b) and (d). This efficiency improvement is achieved with a reduction of 87.2 and $70.6 \%$ on the actualization count $(A C O)$, when compared with the times of updating the Jacobian matrix of the first procedure P1.

Figure 12 presents the performance of the PCPF for the 638-bus and 787-bus systems, solving the same problems of Figure 11 with quadruplicate step size, i.e., 0.008 .
Figure 12(e) and (f) shows the number of iterations and corresponding CPU times considering the step size of 0.012 for the continuation parameter $\alpha$, for the 787-bus system. From the results, it can be concluded that even larger step sizes can be used without affecting the performance of the method during the tracing of the $P-V$ curve. Furthermore, the robustness and computational efficiency improvement are still maintained.

\section{CONCLUSION}

This paper presents a new geometric parameterization technique for the continuation method, which allows not only to obtain the MLP and, subsequently, assessment of voltage stability margin of power systems, but also to obtain the solution points in its vicinity with a low number of iterations. The proposed parameterization technique was developed from the geometrical analysis of the solutions trajectories of the PF equations and it is based on the quadratic characteristic of the curve of total real power losses as a function of loading factor, which presents a similar behavior (curvature) for all operating conditions and electric power systems analyzed.

The parameterization technique eliminates the Jacobian matrix singularity of load flow and therefore all the consequent problems of ill-conditioning as well. This is done by the addition of a second degree equation, i.e., a quadratic function that passes through three points in the plane determined by the loading factor and total real power losses. By using the total real power losses values, normalized by its base case value, the values of both variables loading factor and total real power losses remain within the same range of numerical values. This greatly facilitates the definition of a simple procedure that is able to trace the $P-V$ curve without the need of changing the parameter close to the MLP.

A comparison between the proposed parameterization technique and the ones parameterized by the total real power losses, by the loading factor, and by the bus voltage magnitude, in terms of numbers of iterations is provided. For large-scale real power system, it was shown that the proposed technique presents the best performance. Also, it shows a very attractive option and easy implemented, since it would require few changes in the $\mathrm{CPF}$ model.

The results show that it is also possible to achieve a reduction of computational time (average of 50.8\%) and efficiency improvement in the proposed technique by considering a simple procedure of updating the Jacobian matrix, which is only done when the system undergoes a significant change (changes in the system's topology), without losing robustness. As a future scope the application of the method as an alternative tools for determination of the post-contingency load margins and even to obtain the list of critical contingencies. 


\section{FUNDING}

The authors acknowledge the Brazilian Research Funding Agencies CNPq and CAPES for the financial support.

\section{REFERENCES}

[1] M. Abbott, "Electricity reform and gains from the reallocation of resources," Electr. J., vol. 20, pp. 72-78, 2007.

[2] V. Ajjarapu and C. Christy, "The continuation power flow: A tool for steady state voltage stability analysis," IEEE Trans. Power Systems, vol. 7, no. 1, pp. 416-423, Feb. 1992.

[3] H. D. Chiang, A. J. Flueck, K. S. Shah, and N. Balu, "CPFLOW: A practical tool for tracing power system steady-state stationary behavior due to load and generation variations," IEEE Trans. Power Syst., vol. 10, no. 2, pp. 623-634, May 1995.

[4] D. A. Alves, L. C. P. Da Silva, C. A. Castro, and V. F. Da Costa, "Study of alternative schemes for the parameterization step of the continuation power flow method based on physical parameters, Part I: Mathematical modeling," Electr. Power Compon. Syst.s, vol. 31, pp. 1151-1166, 2003

[5] D. A. Alves, L. C. P. Da Silva, C. A. Castro, and V. F. Da Costa, "Study of alternative schemes for the parameterization step of the continuation power flow method based on physical parameters, Part II: Performance evaluation," Electr. Power Compon. Syst., vol. 31, no. 12, pp. 1167-1177, 2003

[6] E. Garbelini, et al., "An efficient geometric parameterization technique for the continuation power flow," Electr. Power Syst. Res., vol. 77, pp. 71-82, 2007.

[7] Y. Mansour, "Suggested techniques for voltage stability analysis," IEEE Power Eng. Subcommittee, Report 93TH0620-5PWR, 1993.

[8] A. Bonini Neto and D. A. Alves, "An improved parameterization technique for the continuation power flow," in Proc. IEEE PES Transm. Distrib. Conf. Expo., New Orleans, LA, USA, 2010, pp. 1-6.

[9] E. M. Magalhães, A. Bonini Neto, and D. A. Alves, "A parameterization technique for the continuation power flow developed from the analysis of power flow curves," Math. Problems Eng., vol. 2012, pp. 1-24, 2012.

[10] J. Zhao and B. Zhang, "Reasons and countermeasures for computation failures of continuation power flow," in Proc. IEEE Power Eng. Soc. Gen. meet., 2006, p. 6.

[11] A. Bonini Neto and D. A. Alves, "Improved geometric parameterization techniques for continuation power flow," IET Gener., Transmiss. Distrib., vol. 4, pp. 1349-1359, 2010.

[12] H. Sheng and H. D. Chiang, "CDFLOW: Practical tool for tracing stationary behaviors of general distribution networks," IEEE Trans. Power Syst., vol. 29, no. 3, pp. 1365-1371, May 2014.

[13] M. Abdel-Akher, "Voltage stability analysis of unbalanced distribution systems using backward/forward sweep load-flow analysis method with secant predictor," IET Gener., Transmiss. Distrib., vol. 7, no. 3, pp. 309-317, 2013.
[14] Z. Jia and B. Jeyasurya, "Contingency ranking for on-line voltage stability assessment," IEEE Trans. Power Syst., vol. 15, no. 3, pp. 1093-1097, Aug. 2000.

[15] J. Zhao, H. D. Chiang, and H. Li, "Enhanced look-ahead load margin estimation for voltage security assessment," Int. J. Electr. Power Energy Syst., vol. 26, no. 6, pp. 431-438, 2004.

[16] A. Pama and G. Radman, "A new approach for estimating voltage collapse point based on quadratic approximation of PV curves," Electr. Power Syst. Res., vol. 79, pp. 653-659, 2009.

[17] T. Kojima and H. Mori, "Development of nonlinear predictor with a set of predicted points for continuation power flow," Electr. Eng. Japan, vol. 163, no. 4, pp. 30-41, 2008.

[18] S. H. Li and H. D. Chiang, "Nonlinear predictors and hybrid corrector for fast continuation power flow, "IET Gener., Transmiss. Distrib., vol. 2, pp. 341-354, 2008.

[19] PSTCA, Power systems test case archive, 2017. [Online]. Available: https://www2.ee.washington.edu/research/pstca/

[20] E. Kreyszig, Advanced Engineering Mathematics, 7th ed. New York, NY, USA: Wiley, 1993.

[21] A. Semlyen and F. Léon, "Quasi-Newton power flow using partial Jacobian updates," IEEE Trans. Power Syst., vol. 16, no. 3, pp. 332-339, Aug. 2001. doi:10.1109/59.932265.

[22] A. Bonini Neto, E. M. Magalhães, and D. A. Alves, "Dishonest newton method applied in continuation power flow through a geometric parameterization technique," Rev. IEEE Amér. Latina, vol. 14, pp. 161-170, 2016.

\section{BIOGRAPHIES}

Alfredo Bonini Neto received the B.S. degree in mathematics from UNIFADRA, Brazil, in 2002, and the M.S. and Ph.D. degrees in electrical engineering from UNESP in 2006 and 2011, respectively. Since 2014, he has been with São Paulo State University - UNESP, where he is currently an Assistant Professor. His research interests include the areas of mathematic and parameterization techniques applied to continuation power flow.

Elisabete de Mello Magalhães received the B.S. degree in mathematics from UFMS, Brazil, and the M.S. and Ph.D. degrees in electrical engineering from UNESP in 2011 and 2015, respectively. Her research interests include the areas of mathematic and parameterization techniques applied to continuation power flow.

Dilson Amancio Alves received the B.S. degree from USP, Brazil, in 1980, and the M.S. and Ph.D. degrees from UNICAMP in 1988 and 2000, respectively. Since 1991, he has been with UNESP, where he is currently an Assistant Professor. His research interests include static voltage stability analysis. 